\title{
A case for the development of data standards for reporting projections of growth in the Internet's energy consumption
}

\author{
Etienne-Victor Depasquale, Chris Preist \\ Department of Computer Science \\ University of Bristol \\ Bristol, United Kingdom \\ edepa@ieee.org
}

\begin{abstract}
A growing body of literature (the "energy literature"), from academic as well as industrial sources, is contributing to knowledge about the growth of the Internet's energy consumption. Despite a general consensus on trends, there exist significant differences in the values published as well as in the scope of the network under study. A separate body of literature (the "architecture literature") deals with the architecture of the Internet. The architecture literature describes the various segments of the Internet, ranging from the first mile to the transoceanic backbones that link continents. It also describes currentand next-generation architectures of these segments, with emphasis on the first- and second-mile technologies. A rationalisation of the architecture literature is attempted. This is used to facilitate a comparison of a sample of works from the energy literature. Works in this sample forecast energy consumption of metro-area implementations built according to the current-generation architecture, that presents at least two aggregation stages before the level of the network-network-interface at the service provider's core. The rationalisation is presented as a recommendation for authors to facilitate the application of their work as the foundation of research directed towards controlling the Internet's energy consumption.
\end{abstract}

Index Terms-System boundaries, Energy consumption, Internet architecture.

\section{INTRODUCTION}

\section{A. Mutually incoherent energy consumption profiles}

Growth in network energy consumption is widely cited in research that has the objective of controlling this consumption or improving the efficiency of consumption of energy to operate the network. Growth in the metro-core segment (bold and italicised text will be used to indicate that the choice of segment name corresponds to our classification of the segments of the Internet) is predicted to grow at the fastest rate out of all segments [26]. To manage this growth, reliable reporting about actual and predicted consumption is fundamental.

Estimates of energy consumption in large telecommunications networks are available [1] [15] [30] [17]. The availability of several sources should serve to improve identification of the profile of energy consumption by segment of the network. While estimates will differ, it is at least expected that for large networks like the Internet, the profiles would be comparable. It is not expected that major discrepancies arise when comparing the weight of any particular segment among the various sources.

One noteworthy doubt [28] in this regard has in fact been raised: a contrast is made between the claim [17] that the network core will consume as much power (40\%) as the access segment by 2017 and another claim [30] that the "metro/transport and core networks account only for 5 percent" in the same period (2015-2020). This doubt is resolved in our work (Section V-B).

\section{B. Our proposal: standardise segment boundaries}

The difficulty in comparing results of works that set out to assess energy consumption in the global network is well known [1] [2] [3]. In this paper, we address this by first presenting an organisation that attempts to reconcile the boundaries of the global network defined in various works. We then proceed to partition the energy consumption projections along the "harmonised" boundaries and compare the projections where common reference years may be found. In so doing, we identify highest common factors, thereby establishing a base upon which dependent research may be grounded.

The rest of this paper is organised as follows:

- Section II creates the case for harmonised reporting by comparing a sample of the energy literature in their segmentation and terminology;

- Section III suggests some premises in terminology for common elements within the Internet's architecture;

- Section IV suggests a universal method for segmentation of the Internet;

- Section V applies this method to compare the results presented in the chosen sample of the energy literature.

\section{THE CASE FOR HARMONISED REPORTING}

This section shows the architecture of the broadband network found in a sample of three works from the energy literature. The three models are used here to make the case for harmonised reporting. 


\section{A. Ishii et al. [1]}

Fig. 1(b) [1] shows the architecture underlying Ishii et al.'s work. This purports to be a representation of the structure of the broadband network that distributes the Internet in Japan. The segments are presented in bullet form for terseness.

- Access: This comprises the passive optical network (PON). It is rooted in a number of optical line terminals (OLTs) within the telecom vendor's real estate and terminated within customers' real estate in an optical network unit (ONU).

- Aggregation: A ring of switches aggregates/distributes traffic within a zone of a metro area. A number of these rings cover the metro area.

- Metro-core: The edge router represents the IP routing function serving an administrative district of Japan known as a prefecture. The metro-core sub-segment therefore comprises the switching boundary at which IP traffic is either switched to a different metro area within the prefecture or switched to the core network.

- Core: This segment consists of the IP routers that comprise the distribution backbone of the Internet in Japan. Each core router may either switch traffic between edge routers that have a transport connection to it or between an edge router and another core router.

\section{B. Bolla et al. [16]}

Fig. 1(d) [16] is described as a "typical access, metro and core device" network; the legend shows an access node, a transport node and a core node. This architecture is referred to in forecasts of energy consumption in Telecom Italia's broadband network [30]. Both works' citations [16] [30] identify Bolla as the lead author.

- Access: This comprises a set of rings (blue), each of which is the logical topology of the interconnection between "access nodes". The access node is directly connected to customers. Customers' equipment is not shown in Fig. 2.

- Transport: A second set of rings (red) is shown. The caption to this figure [16] refers to "access and metro/core networks" and this same work refers to "transport network nodes". Some equivalence can be deduced between the authors' intentions when referring to "metro" and "transport" segments.

- Core: This segment comprises the inter-metro backbone.

\section{Lange et al. [17]}

Fig. 1(c) [17] shows an "operator's broadband telecommunication network sections."

- Access Network: Several access technologies are included within this segment. Equipment in customers' premises is not included.

- L2 Aggregation: A tree-type, logical layer 2 network is described. The layer 1 hardware is referred to as a "metro/regional" transport network and described as an "underlying optical transport network (OTN)" ring.
- L3 Backbone: This is described as a partly-meshed backbone of IP/MPLS routers, overlying an OTN.

Even at this limited depth of investigation, the summary reveals several differences.

- Ishii includes customer equipment within the access section; Bolla and Lange do not.

- "Aggregation" is used by both Ishii and Lange but not by Bolla.

- Ishii sub-segments the backbone into a metro-core and core. Bolla and Lange do not.

A harmonisation of the boundaries is warranted to facilitate cross-comparison between reports. The harmonisation must include a clear and sufficiently granular analysis of the segments of the network, to justify a fair analysis of these (and other) reports' conclusions.

\section{TERMINOLOGY}

\section{A. Internet architecture}

IETF RFCs such as RFC 4364[4] and RFC 4761[5], as well as the Metro Ethernet Forum's architectural framework [6] (see Section 2 and Appendix II) form part of a collection of standards that employ a consistent terminology to describe components of the Internet's network infrastructure. The formal basis is established in RFC 4026 (e.g. definition of Customer Edge device - CE, Provider Edge device - PE and provider routers that are not attached to CEs - "P routers"), which explicitly addresses the lexical difficulties that arose as providerprovisioned virtual private networks (PPVPNs) were investigated by several research groups. This terminology has been expanded by other RFCs, such as RFC 4761, which defines the user-facing Provider Edge device (u-PE). This scope of application of this terminology has expanded beyond the original scope of PPVPNs into the broader architecture of the Internet. Where possible, similar terminology will be applied here.

\section{B. Providers}

The term "provider" is now a hypernym for organisations characterised by diverse business models. Common labels include "telco", "carrier", "Network Service Providers" (NSPs), "ISP", "content provider", "telecom operator", "network operator", "access provider", "telecoms service provider" and "telecom vendor".

Herzog provides a good rationalisation of the historical development of business models [33]. The telco/carrier/telecom operator/network operator/access provider/telecoms service provider has (historically, at least) built and operated the network within the metro area and beyond it. A curious re-use of the term "transport" is applied here. It does not refer to layer 4 of the OSI model. It refers to the bit-pipe infrastructure: the transceivers, cables, amplifiers, roadside cabinets, ducts, poles, real estate and other such elements that form the physical basis through which telecommunication is guided en route from one end to the other. Henceforth, this role will be referred to as the "telecom vendor". This term has been chosen as it reflects the separation between networks and overlying services that is likely to characterise future business models of this role [33]. 
This role provides connectivity at the physical layer in the form of shared (multi-tenant) infrastructure or dedicated infrastructure (e.g. dark fibre between endpoints). It also provides connectivity at the link layer in the form of virtual private networks (e.g. Metro Ethernet over WDM).

The ISP and the content provider consume the network infrastructure to provide services from OSI layer 3 upward. The NSP may be considered as an older label for that which ISP represents today. NSP is particularly representative of what is considered a Tier 1 ISP. The ISP and the content provider are either wholesale customers of the telecom vendor or are part of the telecom vendor's service set. The ISP and the content provider also consume data centre infrastructure provided by "Internet Exchange Providers", who may be "carrier-neutral" or be part of a "carrier's" set of services. The term carrier is used here as this is the familiar one; "telecom vendor" would be consistent with the choice made in the preceding paragraph. This data centre infrastructure is a point of convergence for interconnection between telecom vendors (intra-group), between ISPs (intra-group) and between content providers and telecom vendors and ISPs (inter-group). The term "Internet Exchange", commonly abbreviated as IX, is the current form of what used to be called the Network Access Point (NAP).

\section{ORGANISATION OF INTERNET NETWORK INFRASTRUCTURE}

\section{A. The Access Segment}

The access segment is the extent of the network that spans from the subscriber's premises to the provider's premises known variously as a Distribution Hub or Local Exchange (LE). Between the two end points, an important intermediate point in the architecture and distribution is the Remote Node (RN).

The active equipment that terminates this segment at the customer's end is commonly referred to as the Customer Premises Equipment (CPE). Within the terminology framework loosely identified in sub-section III-A, the active equipment is referred to as the Customer Edge device (CE). At the Distribution Hub/LE, a user-access convergence device (aka: userfacing provider edge - u-PE) terminates the link. Examples of u-PEs include the digital subscriber loop access multiplexer (DSLAM), the cable modem termination system CMTS), the Optical Line Terminal (OLT) and the NodeB. The latter, of course, is in the field.

The RN is located at kerbs and pavements, where it may be housed in a floor-mounted cabinet, in an enclosure on a pole or inside a manhole. It may serve as a demarcation point in the access segment; for e.g., in [7], the access segment is divided into a Secondary Access part and a Primary Access part, with the RN dividing the two parts. Secondary Access network technologies include wireline PON, VDSL, DOCSIS, UMTS and LTE. Secondary Access is commonly referred to as the last mile (or, conversely, as the first mile, from the customer's perspective) and, as indicated, is demarcated at one end by subscribers' premises and at the other end by a roadside cabinet or pole-mounted enclosure. The components of Secondary Access may be found at various locations along the last mile, starting at the subscriber's end, proceeding through pathways towards roadside cabinets and roadside pole enclosures. The subscriber's end houses CPEs such as ONUs and CMs. The pathways include cabling ducts and pole-spans (overhead). The contents of the RN depend upon the mix-and-match of technologies that comprise the access segment. The roadside cabinet/enclosure may either host a u-PE, or it may host equipment that carries out a physical layer function to split the medium to serve a collection of cable runs to subscribers' premises. An example of the former would be a VDSL2 DSLAM; examples of the latter would be, respectively, (a) a GPON splitter, (b) a DOCSIS HFC optical node and (c) patch panels in ADSL/2 networks.

The Primary Access part spans from the cabinet/enclosure (wireline) or NodeB/BTS site (wireless) to the access network operator's LE. The Primary Access part's technology may either be the same as the Secondary Access part's technology or it may be independent of it. As examples of the former case (a) CMTSs and (b) DSLAMs (ADSL) reach from the Distribution Hub/LE to the CP. As examples of the latter, (a) VDSL2 DSLAMs may uplink to Metro Ethernet aggregation switches over LX/LH or ZX GE and (b) radio access network (RAN) NodeBs backhaul over PONs like ITU-T G.984 (GPON) or IEEE 802.3ah (GE-PON). The upper boundary of the access segment lies at the network-facing interface of the $u-P E$ device.

"Backhaul" is a term that is commonly used with reference to aggregation of individual subscribers' traffic on the access segment. The common interpretation of backhaul considers this aggregation to proceed as far as the boundary with the metrocore segment (see sub-section IV-C) of the network. This boundary is demarcated within the real estate housing the "Metro PoP".

In everyday, popular use, the access network operator is often referred to as a Broadband Service Provider. In section III$\mathrm{B}$, the term "access provider" was shown to refer to these providers. Such providers may provide access to the Internet or may be limited only to operation of the network on behalf of the ISP.

1) Deviations: Demarcation of the access segment

One major disagreement in demarcation of the access segment in literature regards the collector ring that physically interconnects the Distribution Hubs/LEs. In [8], this ring is considered as part of the backhaul network. There is no disagreement perceptible in this but this source proceeds to denote this ring as a metro access/backhaul, implying that the collector ring is part of the access segment. [9] is in tacit agreement: reference is made to a "metro-access ring". In [10], the same source clarifies its understanding of the extent of the access network by graphically mapping it out in the context of a global network. It is a re-affirmation of a notion of the access segment as one that extends beyond the confines of the first major section of real estate, such as the LE or the Distribution Hub. Further affirmation of this understanding is found in [11] (Fig. 2). This view of the network architecture is illustrated in Fig. 3 [8], showing a hierarchy of rings, ending at the metro-core's (see section IV-C) boundary with the Internet's core. A de- 
ployment using current-generation technologies is shown in Fig. 4 [8].

2) Deviations: Demarcation of Primary and Secondary Access

Some access network distributions do not fit cleanly into the primary access-secondary access partition scheme. PONs that include large residential units (like apartment blocks) and large enterprises within their geographical reach may deploy splitters within the building complex or within a private services facility. In such a case, there is no intermediate demarcation between the Distribution Hub/LE and the customer's premises.

P2P optical networks do not manifest a partitioning of the access network. Cables run from a local office, which may be no larger than a shed, directly towards customers' premises. There is not even a user-aggregation device in such P2P access networks. These access networks may be complement by PON deployments to reflect product strategy.

The partitioning scheme is also disrupted by nextgeneration optical networks that reduce or eliminate the need for the LE by exploiting long-reach optical technology to distribute directly from the Central Office (CO). The position of the Optical Line Terminal changes in these next generation networks. Whereas current generation OLTs for GPON and GE-PON distribute fibre from LEs and reach the kerb or the home, next-generation OLTs for WDM-PONs distribute fibre from the CO. See Fig. 5 [8].

\section{B. The metro-aggregation segment}

\section{1) The proposed boundaries}

Proceeding "upward" from the access segment, the metro area network commonly comprises a set of u-PEs [5] (aggregation devices) and one or more Provider Edge aggregation switches. The u-PEs are housed in LEs that cover a carrier's Service Delivery Area. The LEs' traffic is backhauled over a collector ring to the Metro PoP housing one or more aggregation switches (Fig. 4). The u-PEs include devices like ITU-T G.984.x / 1GE / 10GE OLTs, DOCSIS/EuroDOCSIS CMTS and DSLAMs. The aggregation switches include Metro Ethernet switches that aggregate traffic from several u-PE Layer 2 devices. These constitute the means of aggregation of the traffic of a number of access network divisions. The PE aggregation switches and the u-PEs minimally function as L2 devices but may also have limited L3 functionality [5]. The distribution of service edges outside the core and into the metro area of the Internet, in efforts to reduce energy consumption and improve QoS, creates new use cases for L3 connectivity between the CE devices and this segment. An illustration of the role of the aggregation switch is shown in Cisco marketing literature [12]. A comparison with Fig. 4 shows good agreement between these two sources' segmentation of the metro area network, despite differences in terminology (arising out of the different perspectives from which these illustrations were drawn). The lower boundary of the metro-aggregation segment lies at the interface between the u-PEs and the aggregation switches.

The aggregation switches are themselves commonly interconnected in a ring topology (see Fig. 3,4) to two or more
"Edge Routers" (PEs), housed in Metro PoP real estate. The upper boundary of the metro-aggregation segment lies at the interface between the aggregation switch and the transport ring on which the PE router also has an interface.

The bases of the indicated choice of boundaries are two. Firstly, the partitioning is congruous with the intended applications of the technologies referred to. Secondly, a number of works have partitioned in a manner that bears a reasonable similarity to that described hitherto. Fig. 1 cross-references some of these works, using the segment labels that are proposed here. Fig. 1(b) [1] refers to an "aggregation" segment; this segment matches our use of "metro-aggregation" well. Fig. 1(c) [17] makes practically identical use of the term. Fig. 1(d) [16] is a good example of the lack of consistency that this work addresses. There is no reference to an aggregation segment yet inspection of the underlying work reveals that this is the collector ring gathering traffic from the u-PEs. This ring, therefore, is the ring of LEs.

The illustrations included in Fig. 1 manifest some deviations from the reference architecture which we are sketching in this work. The deviation in Fig. 1(d) has been highlighted.

The Metro PoP may also contain the boundary of a local broadband service provider's network. In this case, the Metro PoP may also contain PE routers used in the provision of Virtual Private Line Service (E-Line) and Virtual Private LAN Service (E-LAN).

2) An unfortunate choice of terminology: "aggregation"

The term "aggregation" has been used with reference to collection of traffic from subscribers by u-PEs, collection of traffic from $\mathrm{u}-\mathrm{PEs}$ by $\mathrm{PE}$ aggregation switches and may be used to refer to collection of traffic from $\mathrm{PE}$ aggregation switches into another stage of link-layer aggregation switches (see "Aggr. 2" in Fig. 3). The term "backhaul" is also used to refer to this act of collection of traffic from multiple L2 links onto fewer links having a higher-bandwidth than those "lower" in the hierarchy. "Backhaul" is also interpreted diversely, with some definitions applying this as far back as the core of the network. See, for example, the note in [120, p.4].

Some sources dispense entirely with references to the metro-aggregation segment; see Figs. 2-5. Another source [14] includes the segment in its description of the metro-area network, yet its boundaries lack crisp definition. A publication complementary to this source [15] manifests the same blur. Two distinct segments - "Access and Aggregation" and "Metro" - are presented. The term "Access and Aggregation" is itself inappropriate and no substantial justification is given for the choice of words. The description of what comprises the "Metro" segment compares well with the contents of the metroaggregation segment, despite the lack of architectural detail. Indeed, [7] refers to a "Metro" segment and the description given also compares well with the metro-aggregation segment.

In conclusion, this segment has been identified by no less than the following names: "metro access" [8][9]; "backhaul" [8] or part thereof (as indicated by [13]); part of "access" [16] [10]; "collector" [11] [22, p.153] and "metro collector" [22, p.170]; "metro" [15]; "metro-aggregation" [14] [1] ! We are settling on metro-aggregation and have attempted to describe 
its contents in a manner that facilitates classification of technologies and minor architectural variations.

\section{The metro-core segment}

\section{1) Physical: Real estate and Topology}

The metro-core segment connects a number of Metro PoPs and one or more Core PoPs per metro area. Physical topology of interconnection is commonly a ring [8] [11] [21] [22, p.145, p.152] [24, p.157]; for example, a DWDM ring, installed in $1+1$ redundancy for protection, may link the Metro PoPs to the Core $\mathrm{PoP}(\mathrm{s})[8]$. An illustration of such a topology is shown in Fig. 4, where a metro core DWDM 1+1 ring is shown in the context of a metro-area deployment. The metro-core is also referred to as the metro-regional segment.

Consolidation of ownership of the capital goods comprising the segment varies. At one end, all such goods might be owned by a single operator. The operator would own the metro-core ring transport layer (OSI Layer 1) hardware as well as the premises hosting the Metro PoPs and the Core PoP. Such is the case of Telecom Italia's metro-area network in several Italian cities, interconnecting DSLAMs at the access end to the national backbone at the Long-haul Core end [8, p.360]. At the other end of the range of consolidation of ownership, high fragmentation is found. One operator would own the transport ring hardware. The Metro PoPs might be located in carrierneutral exchanges / colocation centres, where network providers connect to their wholesale clients like ISPs. The Core PoP may be hosted in a carrier-neutral data centre serving as an Internet Exchange for the ISPs in the metro area [18]. The first end of the range corresponds to the cases of the vertically integrated telecommunications provider. Such an operator would occupy Open Access network scenarios (a) - (c) [25]. The other end is closer to case (f) [25]. Open Access design drives towards the interoperability symbolised in case (f).

The metro-core segment is the network that links the carriers that cover the same metro area. It is also the network that interfaces to both the metro-aggregation segment and the core segment. The metro-aggregation segment appears as several "metro-edge" (another term!) rings that are interconnected with the backbone network in Fig. 6 [adapted from 24, p.158].

Smaller metro areas served by very few ISPs may not have a metro core ring and a Core PoP at all. These ISPs might peer directly. The physical location may perhaps consist of real estate adjunct to one of the peers' hosting location. Such an arrangement establishes peering connectivity without granting reciprocal access to premises hosting closely guarded infrastructure.

\section{2) Logical: Traffic flow}

This segment accumulates traffic from the provider(s)'s points of presence within the metro area (Metro PoPs); conversely, it distributes traffic to these Metro PoPs. Traffic flows vertically between any Metro PoP and any Core PoP. Figure 7 [19] illustrates the (logical) relationship between individual ISPs' Metro PoPs and the Core PoP. Traffic also flows between Metro PoPs.

The flow is characterised as meshed (see Fig. 2 (the part labelled (b)) [11] [22, p.148]. The meshing is accomplished through the use of optical add-drop multiplexers (OADMs) at each node of the ring. By passing through an OADM, a lightpath of a given wavelength renders the node transparent and forms a logical connection between the node of insertion (add) and the node of removal (drop). The upper boundary of the metro-core segment lies inside the Core PoP, at the transport interface(s) between the P-routers and the metrocore fibre. This explicitly excludes Core PoP P-routers from the metro-core segment and establishes their transport interface to the metro-core fibre as the boundary between the metro-core and the long-haul (LH) core.

\section{3) Functionality}

The traditional functionality of this segment has been twofold. One function is that of extending the geographical reach of the network to cover longer distances than those possible with the technologies used in the access and metro-aggregation segments. The segment bridges the access and aggregation segments to the long-haul backbone network [22, p.152]. The logic of the division of reach includes the important purpose of reduction of network node complexity. Nodes on shorter links have fewer functional requirements and are less costly to deploy and operate. The second function is that of IP routing. The purpose here is not to delve into the relationship between IP as a client of an underlying transport layer such as DWDM with OADMs at the nodes. The purpose is to identify this layer as that in which IP routing between intra-metro endpoints takes place.

The term metro-core is changing under the pressures of traffic growth [26] and this leads to difficulty in reconciling some works with others. As presented this far, the metro-core segment may be viewed as a segment that aggregates/distributes traffic between the long-haul backbone segment and the metro-aggregation segment (inter-metro), as well as routing traffic within the metro. This is not universally true. Some metro networks have limited or no intra-metro switching capability [23]. Direct reference to the routing function is omitted from the metro network in a well-cited work [17] as late as 2009. In that work, the segment's function seems to be included within the "L3 backbone" segment as there is no reference to the L3 function outside that. In a recent (2014) article [20] published in Alcatel-Lucent's TechZine, arguments are made in favour of "introducing a metro core into the metro aggregation network". The benefits identified may be summarised as one principal benefit: reduction of the length of the path between source and destination. Since traffic flows now increasingly have a source and destination within the metro area, then a routing core capable of switching all such traffic should be part of the metro area network. Figure 8 [20] shows the stage of insertion of the routing. Figure 9 [26] shows the location within the broader context of the metro area network. This graphic amply demonstrates the difference between the view that delegates the routing function to the long-haul backbone [17] [20] [26] and the view that includes it within the metro-core [11] [22]. 


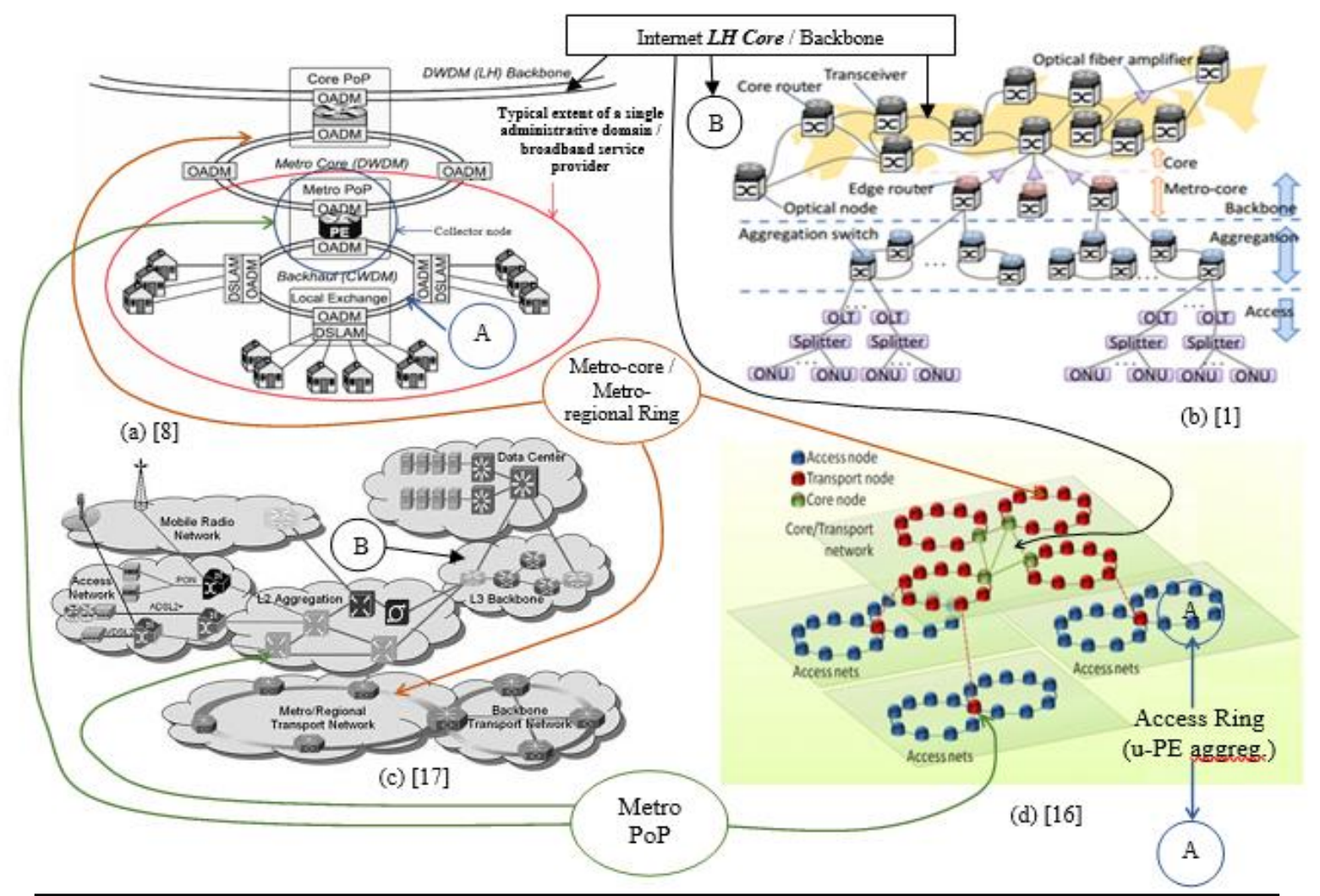

Fig. 1 (a) - (d). Reconciling sources describing the architecture of Internet network infrastructure

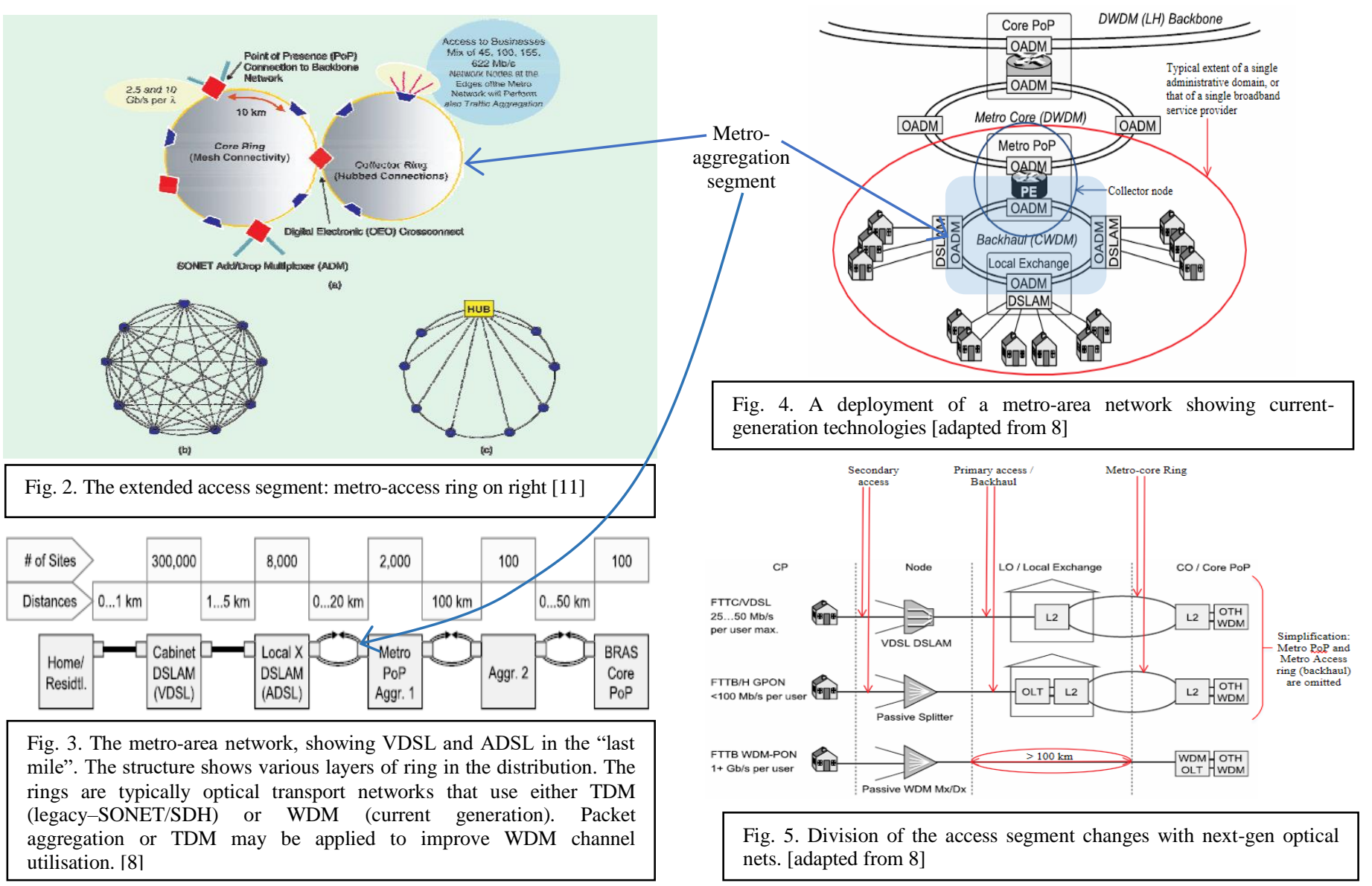


In the former, the metro-core does not exist as a separate segment; in the latter, it is a segment that affords meshed logical connectivity albeit over a physical ring topology. The view in which the metro-core does not exist as a separate segment but rather is integrated within the backbone will be referred to as the first view. The second view, conversely, is that which considers the metro-core as a segment that supports richly distributed (meshed) connectivity between its nodes.

The metro-core router in Fig. 9 [26] seems to have a strikingly similar role to that of the group of routers shown in Fig. 7 [18] inside the Internet Exchange. The resemblance is not coincidental. Their roles are indeed similar. The difference lies in the consolidation implicit in the ownership of the architecture. Both the sources quoted earlier [17] [26] (the latter being the source of Fig. 9) that seem to ignore the existence of a separate metro-core segment relate to vertically integrated operators, whereas Fig. 7 is clearly exhibiting higher degrees of openness according to the Open Access Network set of scenarios. In the circumstance of the vertically-integrated operator, both these sources [17] [26] classify the metro-core router as part of the metro area infrastructure but from the perspective of the ISPs in the multi-player ecosystem shown in Fig.7, the metro presence ends at the Metro PoP. This rationale is confirmed [26] by consideration of the use of a particular integrated-services model of metro-core router in an Internet Exchange application as beyond the scope of a metro-core deployment.

The switching of intra-metro endpoints' traffic away from the Long-haul Core segment may be thought of as a functional description of the metro-core segment. It is achieved through the insertion of routing hardware between the metroaggregation and Long-haul Core segments. Note that the functional description of the metro-core segment shows that notwithstanding the absence of a Core PoP, smaller metro areas can still benefit by establishing the functionality of this segment.

This section has been written to bring the system boundaries into sharp relief as they are essential to a good understanding of trends in energy consumption associated with the transmission, transport, switching and routing of traffic. "The Lexicon is Important" [19]. The illustration in Fig. 10 attempts to facilitate understanding of this section.

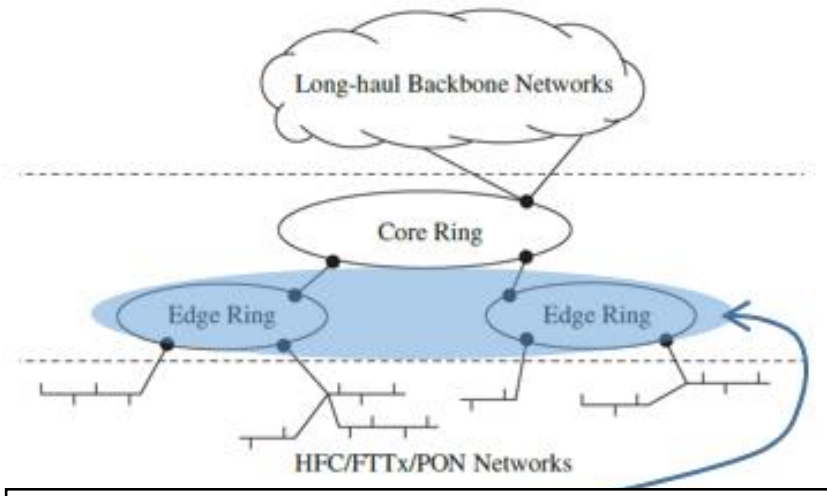

Fig. 6. Multiple rings in the metro-aggregation segment connect to the metro-core ring [adapted from 24, p.158]

\section{COMPARISON OF FOUR REPORTS OF INTERNET ENERGY CONSUMPTION}

This section first analyses the segmentation proposed by the energy literature, then cross-compares their numerical results to the extent permitted by the mutual alignment of the segments. A summary of the segmentation analysis is shown in Table 1. Braces refer to the authors' terms. A summary of the comparison of their numerical results (where relevant, as not all works estimate energy consumption totals) is shown in Table 2.

\section{A. Analysis of segmentation proposed in the energy literature}

\section{1) Internet in Japan [1]}

The authors propose three primary segments: access, aggregation and backbone, the latter divided into metro-core and core.

\section{a) Access $=$ access + CE devices}

The boundary is shown as the network-facing interface of the OLT. This boundary matches the definition proposed in IIB. However, the authors' use of "access" segment includes ONUs; therefore, the comparison identifies this mismatch.

\section{b) Aggregation = metro-aggregation + metro-core}

A single ring of aggregation switches is shown. This architecture matches that shown in Fig. 5. Figure 5 shows that a simplification has been made; the Metro PoP and Metro Access rings (metro aggregation) have been omitted. Therefore, it is immediately visible that some compromise must be made to match this segment with one or other of metro-aggregation and metro-core. There is no clean fit. The logical position of the "edge router" indicated in Fig 1(b) [1] does not fit that of the PE-router. The "edge router" links to other core routers using an erbium-doped fiber amplifier (EDFA)); a PE-router connects to a LH-core router intra-Core-PoP, without such amplification. The most reasonable match would be to place this "aggregation" segment in the same class as the metroaggregation and metro-core segments proposed here and locate the "metro-core" inside the $\boldsymbol{L H}$ core. Since the metro-core has no layer 3 functionality in this case, it corresponds to the first view expressed in sub-section IV-C. Interestingly, NTT holds a dominant position in Japan that compares well with that of a vertically-integrated operator. This strengthens the correlation between the first view and the vertically-integrated operator.

\section{c) Backbone $($ core + metro-core $)=$ Long-haul Core}

This segment visibly corresponds to the long-haul core.

\section{2) Internet in Germany: Deutsche Telekom [17]}

\section{a) Access segment $=$ access}

Various access networks are illustrated, e.g. VDSL2, ADSL2+ and PON. In each case, the network-facing side of the $\mathrm{u}-\mathrm{PE}$ is the upper boundary of the segment. Therefore, this matches the definition proposed here of the access segment.

\section{b) L2 Aggregation = metro-aggregation + metro-core}

A distinction is made between client layer and server layer: the optical transport network is depicted as the server for the aggregation technology chosen. Only one ring is shown but the label attached to it ("metro/regional"), as well as the evidently 


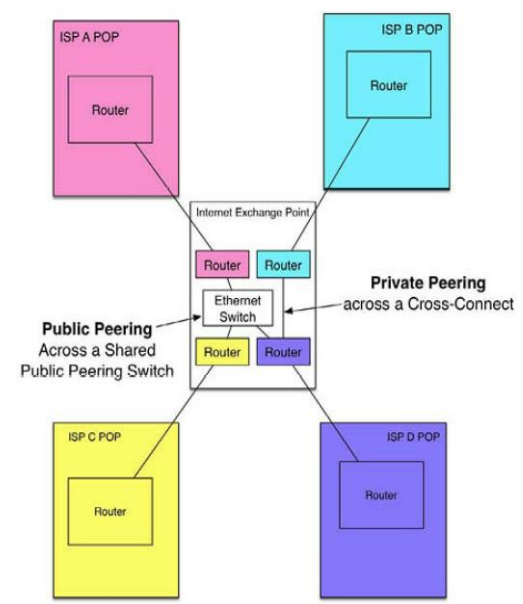

Fig. 7. The metro-core segment comprises a number of Metro PoPs that are logically interconnected at an Internet Exchange that also serves as a Core PoP [graphic taken from $18]$

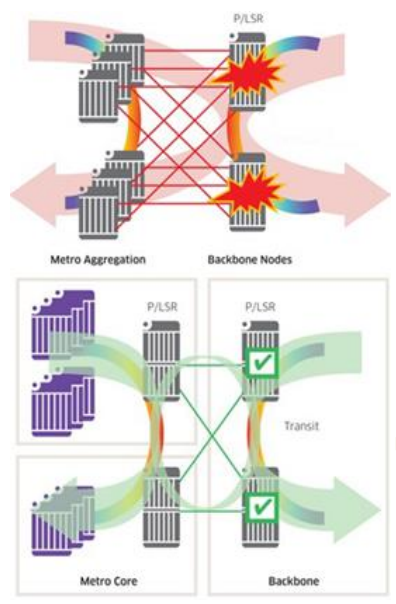

Fig. 8. The metro-core segment introduces routing functionality between the metro-aggregation and Internet backbone. [adapted from 20]
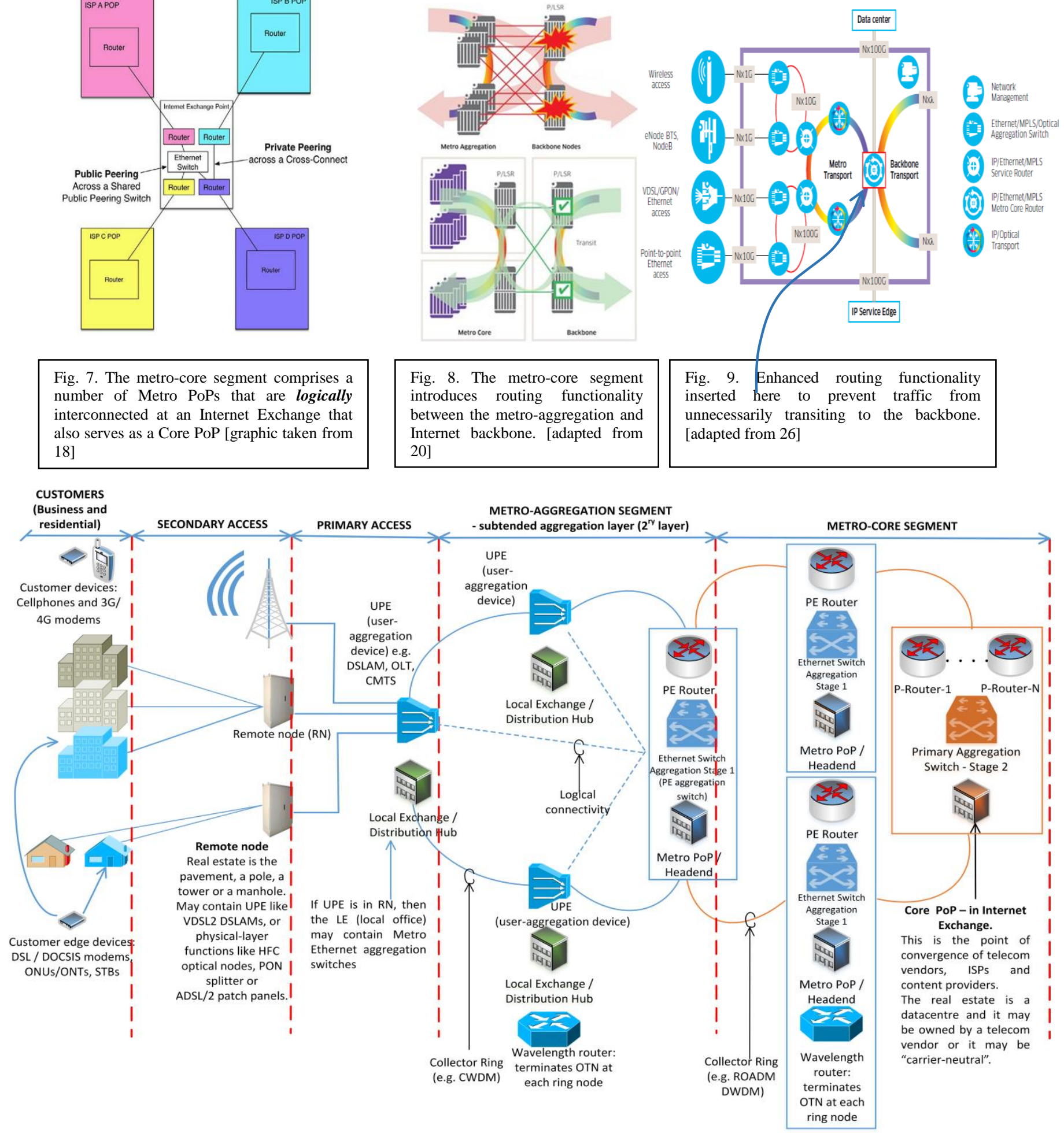

Fig. 10. Illustration complementing the recommended model of segmentation of the metro-area network for analysis and reporting of energy consumption

summative intention of the authors in illustrating this segment (reproduced in Fig. 1(c)) as the intermediary between the u-PEs and the "L3 backbone", leave little room for doubt that this segment best matches the joint metro-aggregation and metrocore segments.
As with Japan's case, Deutsche Telekom is a verticallyintegrated operator. This further strengthens the correlation between the first view and the vertically-integrated operator.

\section{c) L3 Backbone = Long-haul Core}

This segment corresponds to the long-haul core. 


\section{3) Internet in Italy: Telecom Italia [16]}

The boundaries used in [30] are not immediately identifiable as there is no explicit reference within the document to an architecture. Since it is implied that "traffic load values" used in this work are "indicated in other studies", the other study [16] was examined to extract an interpretation. The architecture is shown in Fig. 1(d) [16, Fig.8]. Interpretation is not straightforward as the iconography is basic.

\section{a) Access nets $=$ access + metro-aggregation}

Comparison with Fig. 4 assists the identification of the access nets as the rings that backhaul traffic from the u-PEs. Since the access segment is evidently essential (!) in the metro area network, it is taken to be implicit in "access nets".

\section{b) Transport network = metro-core}

The hierarchical position of the nodes of the transport network, as well as their site at the intersection between two segments, identifies the transport network as the metro-core.

c) Core network = Long-haul core

This segment visibly corresponds to the long-haul core.

4) From the perspective of an early study on energy efficiency of video on demand services

Jayasundara et al. [27] investigated improvements in energy efficiency attainable by moving video caches closer to the point of consumption. While the model [27, Fig.1] does not identify detail about physical topology, it includes sufficient information to justify a comparison with the segments presented here.

"Access" and "core" are readily identifiable with the access and long-haul core segments respectively. Inspection of the model's "metro" segment shows that despite the lack of detail about physical topology, there are two distinct parts to this segment. One part comprises a network between PE routers; the other part comprises an aggregation network that backhauls traffic from the "access" part of the model. Therefore, this model distinguishes between a metro-core and a metroaggregation component but lumps them under the "metro" designation. Despite the superficial similarity with Ishii et al.'s edge router [1], Jayasundara et al.'s model distinguishes itself because it separates the P-router's function from the PErouter's function. Ishii et al. do not evidently distinguish between the long-haul core router, which interfaces to the $\mathrm{LH}$ backbone, and the PE router. The two seem to be lumped. This example illustrates the importance of distinguishing between logical and physical topologies in modelling.

Table I: Comparison of system boundaries with the adopted terminology

\begin{tabular}{|c|c|c|c|c|}
\hline Ref. \# & Access & Metro-Agg. & Metro-core & $\begin{array}{c}\text { Long-haul } \\
\text { Core }\end{array}$ \\
\hline 1 & $\left\{\right.$ Access ${ }^{\text {a }}$ & $\{$ Aggregation $\}$ & $\{$ Aggregation $\}$ & $\{$ Backbone $\}$ \\
\hline 17 & $\{$ Access $\}$ & $\{$ Aggregation $\}$ & $\{$ Aggregation $\}$ & $\{$ Backbone $\}$ \\
\hline 30 & $\{$ Access $\}$ & $\{$ Access $\}$ & $\{$ Metro/Transport $\}$ & $\{$ Core $\}$ \\
\hline 27 & $\{$ Access $\}$ & $\{$ Metro $\}$ & $\{$ Metro & $\{$ Core $\}$ \\
\hline
\end{tabular}

\section{B. Cross-comparison of numerical results: 2017}

The three studies [1] [17] [30] are compared for the year 2017 , which is part of all three studies' estimates. One study
[30] estimates the energy consumption for a five-year period (2015-2020).

A summary of the comparison is shown in Tables II and III. Each study has some differentiators that complicate direct comparison. For example, Japan's operators are planning to shut down use of DSL as FTTH's market share increasingly justifies it [31]. In 2013, Japan's fixed broadband penetration rate $(73 \%)$ into households was substantially higher than Germany's $(64 \%)$ or Italy's (49\%). Furthermore, in the study of Japan's Internet [1], ONUs are included in the access segment calculations; this aggregates the $\mathrm{CE}$ devices consumption inextricably into the access segment's estimate and precludes some comparison [30].

Notwithstanding such difficulties, the noteworthy doubt [28] identified in Section I-A can be resolved. The doubt can be dispelled for two reasons:

1. The figure of $5 \%$ consumption [30] by the network emerges when this is taken relative to the total that includes CE devices, whereas the figure of $40 \%$ [71] excludes it. Indeed, if the CEs are taken into account, the energy consumption of the LH core and metrocore in [17] is estimated to be between $7.3 \%$ and $13.7 \%$ in 2017 . Conversely, if the energy consumption in the CEs is excluded [16], then the percentage of the energy consumption in the metro-core and core (according to the boundary estimations shown in Table 3$)$ is $25.8 \%(=(92+15) /(92+15+307))$.

2. As indicated in [1], the impact of improvements in energy efficiency was not taken into account in [17].

Table II: Percentage of energy consumption found in three different studies for year 2017

\begin{tabular}{|c|c|c|c|c|c|}
\hline $\begin{array}{c}\text { Ref. } \\
\#\end{array}$ & CE & Access & $\begin{array}{c}\text { Metro- } \\
\text { Aggregation }\end{array}$ & $\begin{array}{c}\text { Metro } \\
\text { Core }\end{array}$ & LH Core \\
\hline 1 & 72.8 & 6.5 & 20.7 \\
\hline 17 & 86.3 & \multicolumn{2}{|c|}{6.4} & 7.3 \\
\hline
\end{tabular}

Table III: Percentage of energy consumption found in three different studies for year 2017

\begin{tabular}{|c|c|c|c|c|}
\hline $\begin{array}{c}\text { Ref. } \\
\#\end{array}$ & Access & Metro-Aggregation & $\begin{array}{l}\text { Metro } \\
\text { Core }\end{array}$ & LH Core \\
\hline 17 & 39.3 & \multicolumn{2}{|l|}{21.4} & 39.3 \\
\hline 30 & & 74.2 & 22.2 & 3.6 \\
\hline
\end{tabular}

\section{CONCLUSION AND FUTURE WORK}

In this work, we have recommended the rationalisation of reporting structure and terminology to facilitate crosscomparison between future efforts at measuring and estimating the growth of energy consumption in the Internet. To this end, we have suggested one possible foundation upon which a more detailed reporting framework may be built and a standard developed. We have tried to strengthen our case by drawing attention to the difficulty of cross-comparison where the segments of the architecture either do not include the same set of components or the presence of specific components is ignored.

Such a rationalisation may also be applied to other application domains. For example, there are various sources of traffic estimation, such as Cisco's Visual Networking Index, Sandvine's annual reports and Bell Labs' publications (particu- 
larly in so far as concerns their involvement in GreenTouch). The perspectives of the reports vary. A rationalisation of the various sources may be based upon the same work as that carried out to produce a standard for Internet reporting frameworks.

\section{REFERENCES}

[1] K. Ishii, J. Kurumida, K. i. Sato, T. Kudoh and S. Namiki, "Unifying Top-Down and Bottom-Up Approaches to Evaluate Network Energy Consumption," in Journal of Lightwave Technology, vol. 33, no. 21, pp. 4395-4405, Nov.1, 12015.

[2] V.C. Coroama and L.M. Hilty, "Assessing Internet energy intensity: A review of methods and results", in Environmental Impact Assessment Review, vol. 45, pp. 63-68, 2014.

[3] D. Schien and C. Preist, "A review of top-down models of internet network energy intensity", in Proc. ICT for Sustainability, pp. 8794, 2014.

[4] E. Rosen and Y. Rekhter, "BGP/MPLS IP Virtual Private Networks (VPNs)", RFC 4364, DOI 10.17487/RFC4364, February 2006, <http://www.rfc-editor.org/info/rfc4364>.

[5] K. Kompella, Ed., and Y. Rekhter, Ed., "Virtual Private LAN Service (VPLS) Using BGP for Auto-Discovery and Signaling", RFC 4761, DOI 10.17487/RFC4761, January 2007, <http://www.rfc-editor.org/info/rfc4761>.

[6] Metro Ethernet Network Architecture Framework - Part 1: Generic Framework, 1st ed. The Metro Ethernet Forum, 2004 [Online]. Available:

https://www.mef.net/Assets/Technical_Specifications/PDF/MEF_4. pdf. [Accessed: 02- Apr- 2016]

[7] R. Fratini, M. Savi, G. Verticale and M. Tornatore, "Using replicated video servers for $\mathrm{VoD}$ traffic offloading in integrated metro/access networks," Communications (ICC), 2014 IEEE International Conference on, Sydney, NSW, 2014, pp. 3438-3443

[8] K. Grobe, J. Elbers and S. Neidlinger, "Broadband Access Networks", in Broadband Access Networks: Technologies and Deployments, 1st ed., A. Shami, M. Maier and C. Assi, Ed. Springer Science+Business Media, 2009, pp. 353 - 372.

[9] "Gigabit Ethernet CWDM 80km SFP Optical Transceiver | Finisar Corporation", Finisar.com, 2016. [Online]. Available: https://www.finisar.com/optical-transceivers/fwlf15197dxx. [Accessed: 31- Mar- 2016]

[10] Datacom Telecom Network Diagram - Finisar, 1st ed. Finisar, 2015 [Online]. Available: https://www.finisar.com/sites/default/files/resources/datacom_teleco m_diagrams_handout_-_2015.pdf. [Accessed: 03- Apr- 2016]

[11] I. Tomkos, "Metropolitan area optical networks," in IEEE Circuits and Devices Magazine, vol. 19, no. 4, pp. 24-30, July 2003.

[12] Cisco ME 4924-10GE Ethernet Aggregation Switch, 1st ed. Cisco, $2009 \quad$ [Online]. Available: http://www.cisco.com/c/en/us/products/collateral/switches/me-4900series-ethernet-switches/product_data_sheet0900aecd8052f36b.pdf. [Accessed: 04- Apr- 2016]

[13] R. Chundury, "Mobile broadband backhaul: Addressing the challenge," in Planning Backhaul Networks, Ericsson Review, no. 3, pp. 4-9, 2008.

[14] GreenTouch Foundation, (2015, June). Reducing the Net Energy Consumption in Communications Networks by up to $98 \%$ by 2020 .

[15] Gwatt.net, (2015). G.W.A.T.T.: Global 'What if' Analyzer of neTwork energy consumpTion.
[16] R. Bolla, R. Bruschi, F. Davoli and F. Cucchietti, "Energy Efficiency in the Future Internet: A Survey of Existing Approaches and Trends in Energy-Aware Fixed Network Infrastructures," in IEEE Communications Surveys \& Tutorials, vol. 13, no. 2, pp. 223244, Second Quarter 2011.

[17] C. Lange, D. Kosiankowski, R. Weidmann and A. Gladisch, "Energy Consumption of Telecommunication Networks and Related Improvement Options," in IEEE Journal of Selected Topics in Quantum Electronics, vol. 17, no. 2, pp. 285-295, March-April 2011.

[18] "Amsterdam Internet Exchange News", Ams-ix.net, 2012. [Online]. Available: https://ams-ix.net/newsitems/23. [Accessed: 05- Apr2016]

[19] W. Norton, "Internet Transit," in The 2014 Internet peering playbook. 2014

[20] A. Jansen, "The Case for the Metro Core", TechZine, 2014. [Online]. Available: https://techzine.alcatel-lucent.com/case-metro-core. [Accessed: 16- Apr- 2016]

[21] P. Castoldi et al., "Design of Reliable Metro Core Networks," 2007 9th International Conference on Transparent Optical Networks, Rome, 2007, pp. 130-133.

[22] A. Gumaste and T. Antony, DWDM network designs and engineering solutions. Indianapolis, IN: Cisco Press, 2003.

[23] Upscaling the metro - Deploying the 7950 XRS in the metro core, 1 st ed. Alcatel-Lucent, 2016 [Online]. Available: http://resources.alcatel-lucent.com/asset/171550. [Accessed: 1- Feb2016]

[24] M. Maier, Optical switching networks. Cambridge: Cambridge University Press, 2008.

[25] OASE Deliverable 6.1, "Overview of Tools and Methods and Identification of Value Networks", October 2010.

[26] Metro network traffic growth: an architecture impact study, 1st ed. Alcatel-Lucent, 2013 [Online]. Available: http://resources.alcatellucent.com/asset/171568. [Accessed: 25- Apr- 2016]

[27] C. Jayasundara, A. Nirmalathas, E. Wong and C. Chan, "Improving Energy Efficiency of Video on Demand Services," in IEEE/OSA Journal of Optical Communications and Networking, vol. 3, no. 11, pp. 870-880, November 2011.

[28] A. P. Bianzino, C. Chaudet, D. Rossi and J. L. Rougier, "A Survey of Green Networking Research," in IEEE Communications Surveys \& Tutorials, vol. 14, no. 1, pp. 3-20, First Quarter 2012.

[29] C. Lange, "Energy-related Aspects in Backbone Networks," in Proceedings of 35th European Conference on Optical Communication, September 2009.

[30] R. Bolla, F. Davoli, R. Bruschi, K. Christensen, F. Cucchietti and S. Singh, "The potential impact of green technologies in nextgeneration wireline networks: Is there room for energy saving optimization?" in IEEE Communications Magazine, vol. 49, no. 8, pp. 80-86, August 2011.

[31] "2015 Japan - Telecoms, Mobile and Broadband - BuddeComm", Budde.com.au, 2016. [Online]. Available: http://www.budde.com.au/Research/2015-Japan-Telecoms-Mobileand-Broadband.html. [Accessed: 26- Apr- 2016]

[32] "Fixed and mobile broadband: household penetration rate in 2013 | Statistic", Statista, 2016. [Online]. Available: http://www.statista.com/statistics/199192/household-penetration-offixed-and-mobile-broadband-in-selected-countries/. [Accessed: 26Apr- 2016]

[33] A. Herzog, "The coming carrier network infrastructure - a very different landscape." 1st ed. Alcatel Lucent, 2007. 